\title{
The future of force fields in computer-aided drug design
}

\author{
Daniel J Cole*,1 (D), Joshua T Horton ${ }^{1}$, Lauren Nelson ${ }^{1} \&$ Vadiraj Kurdekar ${ }^{1}$ \\ ${ }^{1}$ School of Natural \& Environmental Sciences, Newcastle University, Newcastle upon Tyne, NE1 7RU, UK \\ *Author for correspondence: daniel.cole@ncl.ac.uk
}

\begin{abstract}
"As the popularity and accessible time scales of computer simulations grow, it is becoming apparent that more accurate force fields are required to overcome limitations in predictive molecular design."
\end{abstract}

First draft submitted: 2 July 2019; Accepted for publication: 9 July 2019; Published online: 23 September 2019

Keywords: computational chemistry and molecular modeling $\bullet$ drug discovery $\bullet$ physical chemistry $\bullet$ quantum mechanics • statistical mechanics

Computational molecular simulation has the potential to answer many questions regarding the role of biological molecules in human health and disease, and make predictions about the binding affinity and selectivity of small molecules [1]. Increased access to high performance computing resources and graphical processing units, alongside improvements in sampling protocols, enables computational simulations to routinely provide dynamical information on the nanosecond to millisecond time scale. Such information can provide insight into biological function that is complementary to experimental structural biology techniques, such as $\mathrm{x}$-ray crystallography and nuclear magnetic resonance. In structure-based computer-aided drug design (CADD), virtual screening aims to suggest new drug candidates by docking large libraries of small molecules into the binding site of the therapeutic target and using scoring functions to determine binding poses and estimate binding affinity [2]. While virtual screening is useful for high-throughput studies, inherent approximations limit accuracy, especially when target and/or ligand flexibility are crucial. Statistical thermodynamic methods, such as free energy perturbation (FEP), provide a more rigorous estimate of the protein-ligand binding free energy. Ultimately, the goal of FEP is to screen protein-ligand binding free energies of large numbers of potential drug candidates with sufficient accuracy that it can be used to prioritize compounds for synthesis [3].

\section{Molecular mechanics force fields}

Underlying many of these techniques in structure-based CADD is the molecular mechanics force field, which describes the total energy of the system as a function of the atomic coordinates. The most widely used biological force fields, AMBER, CHARMM and OPLS, share similar functional forms which have remained largely unchanged for many decades [4]. These force fields treat nonbonded interactions as a physically motivated Coulomb interaction between (usually) atom-centered point charges, supplemented by a Lennard-Jones interaction. The latter is repulsive at short interatomic distance to model overlap of electron clouds, and attractive at longer range to account for van der Waals interactions. Covalently bonded atoms are described by harmonic bond-stretching and angle-bending terms, and anharmonic torsional energies. All of these interactions require parameterization. That is, quantities such as equilibrium bond lengths, force constants and atomic charges must be assigned. Parameterization strategies vary but are characterized by extensive fitting to experimental and quantum mechanical (QM) properties of small organic molecules.

As the popularity and accessible time scales of computer simulations grow, it is becoming apparent that more accurate force fields are required to overcome limitations in predictive molecular design. For small molecule validation studies, such as liquid physical properties and host-guest binding, for which sampling requirements can be readily met, the force field is often shown to limit the predictive utility of molecular modeling [5]. Turning to the calculation of protein-ligand binding free energies, it is generally assumed that FEP accuracy of $0.6-1.0 \mathrm{kcal} / \mathrm{mol}$ 
is required to guide medicinal chemistry optimization; an accuracy that is currently only achieved in isolated bestcase scenarios. Protein force fields present particular challenges, such as appropriately balancing protein-protein and protein-water interaction strengths and accounting for coupling between backbone and side chain dynamics (e.g., the optimal amino acid side chain torsion parameters may depend on whether the backbone is in an $\alpha$-helix or $\beta$-sheet conformation). In long timescale simulations of proteins, recent experimental data show that state-of-the-art force fields fail to accurately model intrinsically disordered proteins and temperature-dependent folding [6]. There is evidence that employing residue-specific torsion parameters and retuning the nonbonded interaction parameters of the water model have a strong influence on these computed properties. As we shall argue, however, it may be necessary to more fully understand the underlying physical nature of these interactions rather than employing ad hoc empirical parameter adjustment, which may be masking underlying issues with the computational model.

\section{New approaches to force field parameterization}

A danger associated with incremental, often labor-intensive, force field parameter updates is that accuracy will begin to stagnate. A fundamentally new approach to force field design is required, incorporating up-to-date techniques in quantum chemistry and data science. The Open Force Field Initiative (https://openforcefield.org) has recently highlighted one issue associated with legacy force field atom types, which are used to label atoms in a molecule and then assign their parameters from libraries. These atom types need to be curated by human experts and typically lead to a proliferation of redundant parameters, thus hindering automated force field development. They address this by introducing a new force field format, SMIRNOFF, which assigns parameters based on the full chemical environment of an atom (the SMIRKS pattern) through direct chemical perception [7] rather than going through the intermediate steps of atom typing. In this way, a general organic force field parameter library may be written in less than 350 lines rather than several thousand lines for typical modern force fields. While this does not immediately improve accuracy (though several inconsistencies and instances of human error in legacy force fields did surface), in combination with the open software and data infrastructures for force field design, automated protocols for parameter fitting and best practice property prediction advocated by the Open Force Field Initiative, it provides a very promising starting point for rigorous and automated parameter fitting.

The force field parameter libraries described above are traditionally fit to reproduce the experimental or QM properties of a representative set of small molecules and then applied to molecules outside the training set using atom types (or SMIRKS patterns in the case of SMIRNOFF). These transferable force fields give the user the ability to rapidly parameterize diverse expanses of chemical space at low computational cost. However, there are potential disadvantages to this approach. First, experimental data may be unavailable for many systems of significant physical and chemical interest (for example, organometallic complexes or molecules in electronic excited states). Thus, the traditional approach of fitting suitably accurate force field parameters to experiment is difficult for these molecules. Second, it is well established that electron density (and hence atomic charge) polarizes in response to the atom's environment. For example, Jorgensen $e$ al. have shown that the QM hydrogen bonding interaction between para-substituted phenols and water can vary by around $2 \mathrm{kcal} / \mathrm{mol}$ depending on the nature of the para substituent, which could have important consequences for choices of ring substituents and heterocycles during hit-to-lead optimization [8]. The observed trend could not be captured by a transferable charge set. For this reason, it is commonplace to assign 'bespoke' atomic charges that are computed directly for the small molecule under study. Interestingly, recent advances in quantum chemistry indicate that van der Waals interaction strengths are similarly dependent on the atomic environment. For example, Gobre and Tkatchenko studied the range, and scaling with system size, of the $\mathrm{C}_{6}$ coefficient (a measure of the van der Waals strength) in carbon nanostructures and found that it can range from around 20 atomic units (a.u.) in diamond to 150 a.u. in graphene [9]. The nature of the van der Waals interaction is less commonly studied in medicinal chemistry, but it is clear that much remains to be understood about its role in protein-ligand binding and its treatment in force fields [10].

\section{Molecule-specific force fields}

An alternative to the transferable force fields described above is to instead parameterize the system on a case-by-case basis. By deriving parameters specifically for the system under study, the assumptions concerning charge and van der Waals parameter transferability are reduced. To avoid experimental data curation, such force fields are necessarily derived directly from quantum mechanical $(\mathrm{QM})$ calculations. The earliest QM-derived molecule-specific force fields date back several decades, but with major advances in computational resources, there is now an increasing 
range of automated derivation techniques available [11,12], which is crucial if they are to be used in high-throughput workflows in, for example, computer-aided drug design.

The quantum mechanical bespoke (QUBE) force field for proteins and small molecules is one such moleculespecific force field design protocol, with a particular focus on extensibility to large, heterogeneous systems, such as protein-ligand complexes. QUBE utilizes the modified Seminario method to derive bond and angle parameters from the QM optimized structure and Hessian matrix [13]. The accuracy of the bond and angle parameters may be determined by comparing force field vibrational frequencies with QM, and QUBE is competitive with modern transferable force fields, and offers particular accuracy improvements for more complex molecules containing heterocycles. To facilitate scaling to arbitrarily large system sizes and to treat charge and Lennard-Jones parameter derivation on a consistent footing, all QUBE nonbonded parameters are derived from atoms-in-molecule electron density partitioning of a single QM calculation. QUBE parameter derivation is fully automated through the open source QUBEKit software, and the resulting force fields have been extensively benchmarked against experimental liquid properties, offering similar accuracy to transferable force fields, which have been fit to these data [14]. Following implementation in the ONETEP linear-scaling density functional theory code (www.onetep.org), QUBE nonbonded parameters may be derived for systems comprising many thousands of atoms, including entire proteins. The atomic charges reproduce the QM electrostatic potential of proteins more accurately than transferable force field charges, and they are relatively insensitive to small conformational changes; both of which are crucial requirements for flexible force field design. Torsional parameters are derived from QM dihedral scans, and a library of these parameters has been built for use in protein simulations. A QUBE force field has been derived for the L99A mutant of T4 lysozyme, and been shown to outperform the OPLS-AA/M force field in the prediction of absolute binding free energies of six benzene analogs to the protein $(0.85 \mathrm{vs} 1.26 \mathrm{kcal} / \mathrm{mol}$ mean unsigned errors) [15].

One disadvantage of molecule-specific force fields is of course the time cost associated with the underlying QM calculations. However, a promising way around this is to exploit advances in machine learning to train computer models to predict force field parameters at the desired QM level using only the molecular environment as input. By way of example, Riniker and coworkers have recently built a machine learning model to predict the atomsin-molecule charges of molecules using only the $2 \mathrm{D}$ molecular topology as a descriptor (that is, with no QM calculation) [16]. By training on $130 \mathrm{k}$ lead-like molecules, the model is able to rapidly and accurately predict the atomic charges of a test dataset of drug-like compounds (root mean square error $<0.02 \mathrm{e}$ ).

\section{Beyond the standard functional form}

The ultimate goal of force field development work is to approximate as closely as possible the full solution to the Schrödinger equation at affordable computational expense. Of course, the definition of affordable depends strongly on the problem under investigation, and much work has been done to attempt to break down the QM interaction energy into physically meaningful and calculable components [17]. At the more accurate end of the scale (e.g., symmetry-adapted perturbation theory), the computational expense currently hinders application in medicinal chemistry. On the other hand, polarizable force fields (of which AMOEBA is a well-known example) seek to account for changes in the charge distribution of a molecule in response to the electric field while retaining the ability of the force field to simulate the dynamics of biological systems. Such considerations should be crucial when modeling, for example, the partitioning of a small molecule between a polar solvent and a hydrophobic protein binding site.

However, in general, the addition of extra force field terms can also add to the labor associated with parameterization. Visscher and Geerke address this problem by extending aspects of the quantum mechanically derived force fields described above to include both higher order van der Waals interactions (including $\mathrm{C}_{8}$ interactions) and atomic polarization [18]. This force field model is able to reproduce the liquid densities and heats of vaporization of 11 linear and branched alkanes with rms errors of $0.02 \mathrm{~g} / \mathrm{cm}^{3}$ and $0.15 \mathrm{kcal} / \mathrm{mol}$, respectively, and it will be interesting to see whether this level of accuracy is maintained for more polar biological building blocks.

Finally, we saw above that advances in machine learning may be harnessed to accelerate parameter assignment. Though more commonly employed in the materials sciences, machine learning is now emerging as a tool, not just to extract force field parameters, but to actually learn the underlying QM potential energy surfaces of organic molecules. One such example is the ANI deep neural network potential [19], the first iteration of which was trained on a database of millions of QM calculations, spanning conformational and configurational space of more than $57 \mathrm{k}$ small organic molecules containing $\mathrm{H}, \mathrm{C}, \mathrm{N}$ and $\mathrm{O}$ atoms. The result is, in the authors' words, a potential game changer for molecular simulation - an interatomic potential that is chemically accurate relative to the underlying 
QM calculations, but orders of magnitude faster. Perhaps most importantly, the authors showed that the potential is extensible to molecules much larger than those used for training (up to 54 atoms), which already at this early stage make machine learning potentials an alternative to expensive QM methods for simulating structural properties of drug-like molecules.

\section{Conclusion \& future perspective}

Molecular mechanics force fields are a crucial computational technology, underpinning biological modeling and rational molecular design. Traditional force fields are undergoing continuing improvements, and gains in accuracy are apparent; yet legacy issues may limit the step changes in accuracy that are desired. Here, we have outlined some of the approaches that, in our opinion, will be vital in next generation force field design. Perhaps the most important advance will be to reduce the labor associated with force field parameterization by employing open and automated data curation, parameter fitting and force field validation approaches. As well as the expected accuracy gains, this will allow researchers to standardize parameterization protocols, so that, for example, modified amino acids, lipids and DNA/RNA are treated at the same accuracy as small molecules and proteins, and also to move away from legacy force field functional forms and rapidly investigate new physical models that more accurately capture QM interaction effects (e.g., van der Waals, polarization [20]). Allied with this, it is important to continue to investigate new methods for extracting force field parameters directly from QM. This will not only allow us to derive molecule-specific force fields, which are already showing promise in biomolecular modeling, but will also accelerate transferable force field design by reducing overfitting to experimental data. The natural and exciting progression is machine learning of the full QM potential energy surface, though it will be important to evaluate these potentials in the condensed phase where long-ranged many-body electrostatic effects are crucial and may not be sufficiently represented in small molecule training datasets.

\section{Financial \& competing interests disclosure}

DJ Cole and V Kurdekar are grateful for financial support from EPSRC (grant number EP/R010153/1). The authors have no other relevant affiliations or financial involvement with any organization or entity with a financial interest in or financial conflict with the subject matter or materials discussed in the manuscript apart from those disclosed.

No writing assistance was utilized in the production of this manuscript.

\section{Open access}

This work is licensed under the Creative Commons Attribution 4.0 License. To view a copy of this license, visit https://creativecommons.org/licenses/by/4.0/.

\section{References}

1. Huggins DJ, Biggin PC, Dämgen MA et al. Biomolecular simulations: from dynamics and mechanisms to computational assays of biological activity. Wiley Interdis. Rev. 9(3), e1393 (2019).

2. Gimeno A, Ojeda-Montes MJ, Tomás-Hernández S et al. The light and dark sides of virtual screening: what is there to know? Int. J. Mol. Sci. 20(6), 1375 (2019).

3. Li Z, Huang Y, Wu Y et al. Absolute binding free energy calculation and design of a subnanomolar inhibitor of phosphodiesterase-10. J. Med. Chem. 62(4), 2099-2111 (2019).

4. Jorgensen WL. Foundations of biomolecular modeling. Cell 155(6), 1199-1202 (2013).

5. Yin J, Henriksen NM, Slochower DR et al. Overview of the SAMPL5 host-guest challenge: are we doing better? J. Comput. Aided Mol. Des. 31(1), 1-19 (2017).

6. Nerenberg PS, Head-Gordon T. New developments in force fields for biomolecular simulations. Curr. Opin. Struct. Biol. 49, 129-138 (2018).

7. Mobley DL, Bannan CC, Rizzi A et al. Escaping atom types in force fields using direct chemical perception. J. Chem. Theory Comput. 14(11), 6076-6092 (2018).

8. Jorgensen WL, Jensen KP, Alexandrova AN. Polarization effects for hydrogen-bonded complexes of substituted phenols with water and chloride ion. J. Chem. Theory Comput. 3(6), 1987-1992 (2007).

9. Gobre VV, Tkatchenko A. Scaling laws for van der Waals interactions in nanostructured materials. Nat. Comm. 4, 2341 (2013).

10. Walters ET, Mohebifar M, Johnson ER, Rowley CN. Evaluating the London dispersion coefficients of protein force fields using the exchange-hole dipole moment model. J. Phys. Chem. B. 122(26), 6690-6701 (2018). 
11. Greff da Silveira L, Jacobs M, Prampolini G, Livotto PR, Cacelli I. Development and validation of quantum mechanically derived force-fields: thermodynamic, structural, and vibrational properties of aromatic heterocycles. J. Chem. Theory Comput. 14(9), 4884-4900 (2018).

12. Grimme S. A general quantum mechanically derived force field (QMDFF) for molecules and condensed phase simulations. J. Chem. Theory Comput. 10(10), 4497-4514 (2014).

13. Allen AEA, Payne MC, Cole DJ. Harmonic force constants for molecular mechanics force fields via Hessian matrix projection. J. Chem. Theory Comput. 14(1), 274-281 (2018).

14. Horton JT, Allen AEA, Dodda LS, Cole DJ. QUBEKit: automating the derivation of force field parameters from quantum mechanics. J. Chem. Inf. Model. 59(4), 1366-1381 (2019).

15. Cole DJ, de Vaca IC, Jorgensen WL. Computation of protein-ligand binding free energies using quantum mechanical bespoke force fields. Med. Chem. Commun.10, 1116-1120 (2019).

16. Bleiziffer $P$, Schaller K, Riniker S. Machine learning of partial charges derived from high-quality quantum-mechanical calculations. $J$. Chem. Inf. Model. 58(3), 579-590 (2018).

17. Stone AJ. Intermolecular potentials. Science 321(5890), 787-789 (2008).

18. Visscher KM, Geerke DP. Deriving force-field parameters from first principles using a polarizable and higher order dispersion model. $J$. Chem. Theory Comput. 15(3), 1875-1883 (2019).

19. Smith JS, Isayev O, Roitberg AE. ANI-1: an extensible neural network potential with DFT accuracy at force field computational cost. Chem. Sci. 8(4), 3192-3203 (2017).

20. Manz TA, Chen T, Cole DJ, Limas NG, Fiszbein B. New scaling relationships to compute atom-in-material polarizabilities and dispersion coefficients: part 1. Theory and accuracy. RSC Adv. 9, 19297-19324 (2019). 
